\title{
Naringenin inhibits allergen-induced airway remodeling in a murine model of asthma
}

\author{
YING SHI, YAN TAN, SHAN MAO and WEI GU
}

Department of Respiratory Medicine, Nanjing First Hospital, Nanjing Medical University, Nanjing, Jiangsu 210006, P.R. China

Received June 20, 2013; Accepted January 23, 2014

DOI: $10.3892 / \mathrm{mmr} .2014 .1940$

\begin{abstract}
The flavonoid naringenin has been shown to attenuate airway inflammation and airway hyper-reactivity in acute murine models of asthma. The purpose of this study was to investigate the effects of naringenin in allergen-induced airway remodeling in mice. Ovalbumin (OVA)-sensitized mice were challenged with OVA for 8 weeks to produce a model of chronic asthma. Airway hyper-responsiveness (AHR), inflammation and remodeling were evaluated in mice receiving naringenin prior to OVA challenge. Compared to OVA-sensitized and -challenged mice, those treated with naringenin showed markedly attenuated chronic inflammation, persistent AHR and airway remodeling. In addition, naringenin treatment caused a significant reduction in the levels of total serum $\mathrm{IgE}$ and of T helper 2 (Th2) cytokines in the bronchoalveolar lavage fluid (BALF). Naringenin may thus delay the progression of airway remodeling, providing a potential treatment for asthma.
\end{abstract}

\section{Introduction}

Bronchial asthma is an allergic disease manifesting with symptoms such as wheezing, breathlessness, chest tightness and coughing (1). Cardinal features of the disease are airway hyper-responsiveness (AHR), inflammation and remodeling (2). Airway remodeling refers to structural changes of the airway wall arising from unresolved inflammation, including epithelial denudation, subepithelial fibrosis, mucus gland hypertrophy, myofibroblast and smooth muscle proliferation and angiogenesis (3-5). These structural changes play important roles in the pathophysiology of asthma. In patients with severe asthma, airway remodeling is associated with airway thickening, airway flow limitation and AHR (6).

Naringenin is a naturally occurring flavonoid possessing anti-inflammatory and antiproliferative activities $(7,8)$. We have previously demonstrated that naringenin attenuates acute inflammation and AHR in allergen-induced murine models of

Correspondence to: Professor Wei Gu, Department of Respiratory Medicine, Nanjing First Hospital, Nanjing Medical University, 68 Changle Road, Nanjing, Jiangsu 210006, P.R. China

E-mail:sykxss@126.com

Key words: airway remodeling, asthma, naringenin asthma (9). There are also reports that naringenin can inhibit neointimal hyperplasia and smooth muscle cell proliferation $(10,11)$. Therefore, we hypothesized that naringenin may interfere with the structural changes occurring in chronic airway diseases. In the present study, we investigated the effects of naringenin on airway remodeling in a murine model of asthma.

\section{Materials and methods}

Antigen sensitization, challenge and naringenin treatment. Female BALB/c mice (6-8 weeks old) were purchased from the Shanghai Laboratory Animal, Inc. (Shanghai, China). The experimental procedures involving these mice were approved by the Nanjing Medical University and followed the guidelines of the Institutional Animal Care and Use Committee. Naringenin (Sigma-Aldrich, St. Louis, MO, USA) was dissolved in dimethylsulfoxide to obtain a final concentration of $0.1 \mathrm{mg} / \mu \mathrm{l}(367 \mathrm{mM})$, used as the stock solution.

Mice were randomly divided into four groups according to the difference of antigen in sensitization and treatment before challenge, namely control group, ovalbumin (OVA) group, dexamethasone group and naringenin group. Dexamethasone was purchased from Tianyao Company (Hubei, China) and was used at a concentration of $1 \mathrm{mg} / \mathrm{ml}$. On days 0,7 and 14, mice of the OVA, dexamethasone and naringenin groups were immunosensitized by intraperitoneal injection of $100 \mu \mathrm{g}$ chicken egg OVA (grade V; Sigma-Aldrich) adsorbed to $100 \mu \mathrm{l}$ of Imject Alum adjuvant (Pierce Biotechnology, Inc., Rockford, IL, USA). From day 21, the mice were challenged with $1 \%$ OVA aerosolized for $30 \mathrm{~min} /$ day, 3 days/week for 8 weeks. Mice of the dexamethasone and naringenin groups received intraperitoneal injections of dexamethasone $(5 \mathrm{mg} /$ $\mathrm{kg}$ body weight) and naringenin (50 mg/kg body weight) $1 \mathrm{~h}$ prior to each OVA challenge. The dose of naringenin was selected based on our previous study (9). Mice of the negative control group were sham-sensitized and -challenged using phosphate-buffered saline (PBS).

Airway physiology. Airway responsiveness was measured $24 \mathrm{~h}$ after the last OVA challenge. The mice were anesthetized, tracheostomized and placed in a whole-body plethysmograph (Synol High-Tech, Beijing, China). Then, mice were ventilated via an intratracheal tube at 90 breaths/min. Methacholine was administered through the caudal vein. The maximal values 
of lung resistance were measured after the administration of increasing doses of methacholine $(10-200 \mu \mathrm{g} / \mathrm{kg})$.

Analysis of bronchoalveolar lavage fluid (BALF) and serum. The airway lumina were washed 3 times with $0.5 \mathrm{ml}$ of PBS. The BALF was centrifuged and the upper fluid sample was retained for IL-4 and IL-13 detection. The total number of different cell types in the BALF was estimated using Wright's staining and a haemocytometer; at least 200 cells were counted. Serum IgE levels were measured with the OptEIA ${ }^{\mathrm{TM}}$ ELISA kit (BD Biosciences, San Diego, CA, USA).

Lung histology and periodic acid schiff (PAS) staining. The left lungs, collected $24 \mathrm{~h}$ after the last OVA challenge, were fixed in formalin, embedded in paraffin, sectioned and stained with hematoxylin and eosin solution and PAS. Blind-scoring histological analyses of the four groups was performed by pathologists. To quantify the mucus production in the airways, the number of PAS-negative and -positive epithelial cells (goblet cells) in individual bronchioles was counted as previously described (12). The percentage of PAS-positive cells was calculated as the number of PAS-positive epithelial cells per bronchiole divided by the total number of epithelial cells.

Immunohistochemical detection of $\alpha$-smooth muscle actin $(\alpha-S M A)$. Sections were deparaffinized and rehydrated in graduated alcohol solutions. Endogenous peroxidase activity was blocked by incubation with $3 \% \mathrm{H}_{2} \mathrm{O}_{2}$. Specimens were flooded with $5 \%$ normal goat serum to prevent non-specific absorption of immunoglobulin. Specimens were then incubated with mouse anti-human $\alpha$-SMA moloclonal antibody (Dako, Glostrup, Denmark). For the negative control, the primary antibody was replaced with normal rabbit IgG. The slides were incubated overnight and rinsed with PBS, then incubated with peroxidase-labeled goat anti-mouse IgG (Dako) for $30 \mathrm{~min}$ at $37^{\circ} \mathrm{C}$, washed again with $\mathrm{PBS}$, and stained with diaminobenzidine (Santa Cruz Biotechnology, Inc., Santa Cruz, CA, USA). The sections were counterstained with hematoxylin and eosin, dehydrated and observed under a light microscope. At least 10 bronchioles were counted in each of the slides. Data were expressed as the immunostained $\alpha$-SMA area per length of the basement membrane of bronchioles (internal diameter, $150-200 \mu \mathrm{m}^{2}$ ).

Assessment of peribronchial fibrosis. The total collagen content of the lung was determined according to the protocol of a commercial hydroxyproline detection kit (Nanjing Jiancheng Bioengineering Institute, Nanjing, China). The peribronchial area was stained with Masson's trichrome staining and quantified using a light microscope attached to an image-analysis system (Simple PCI, version 5.2; Leica, Mannheim, Germany). At least 10 bronchioles were counted in each of the slides. Data were expressed as the stained area per length of the basement membrane of bronchioles.

Statistical analysis. Data were presented as means \pm SEM. Statistical comparisons were performed with one-way ANOVA using the SPSS 16.0 software (IBM SPSS Statistics, Armonk, $\mathrm{NY}$, USA). $\mathrm{P}<0.05$ was considered to indicate a statistically significant difference.
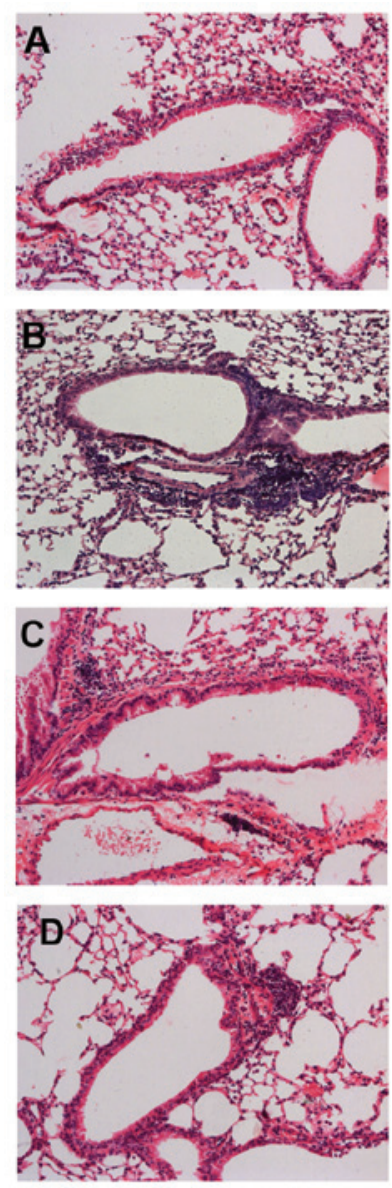

Figure 1. Naringenin attenuates allergic airway inflammation. Images of hematoxylin and eosin staining in the (A) control, (B) ovalbumin, (C) dexamethasone and (D) naringenin groups.

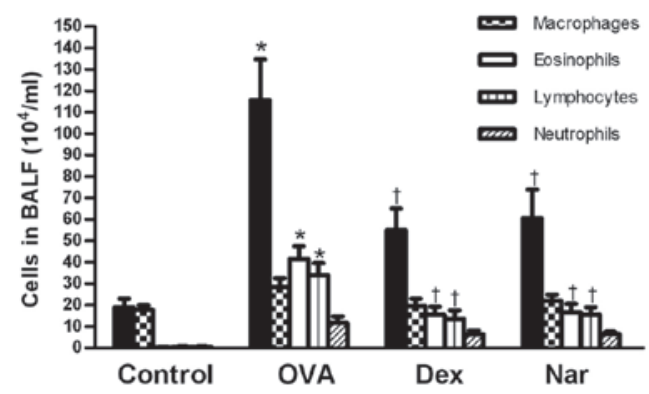

Figure 2. Cell differentiation in bronchoalveolar lavage fluid (BALF). A total of 6 mice were examined in three independent experiments. ${ }^{*} \mathrm{P}<0.05$ between the control and the ovalbumin (OVA) group; ${ }^{\dagger} \mathrm{P}<0.05$ between the OVA and dexamethasone (Dex) or naringenin (Nar) groups.

\section{Results}

Naringenin attenuates chronic airway inflammation. Marked influxes of inflammatory cells into the airway and around the blood vessels were observed in the OVA-sensitized and -challenged mice, but not in the control mice. Mice treated with naringenin and dexamethasone showed marked reductions in the infiltration of inflammatory cells compared to the OVA-treated group (Fig. 1).

Following sensitization and challenge with OVA, the number of total leukocytes, eosinophils and lymphocytes were 


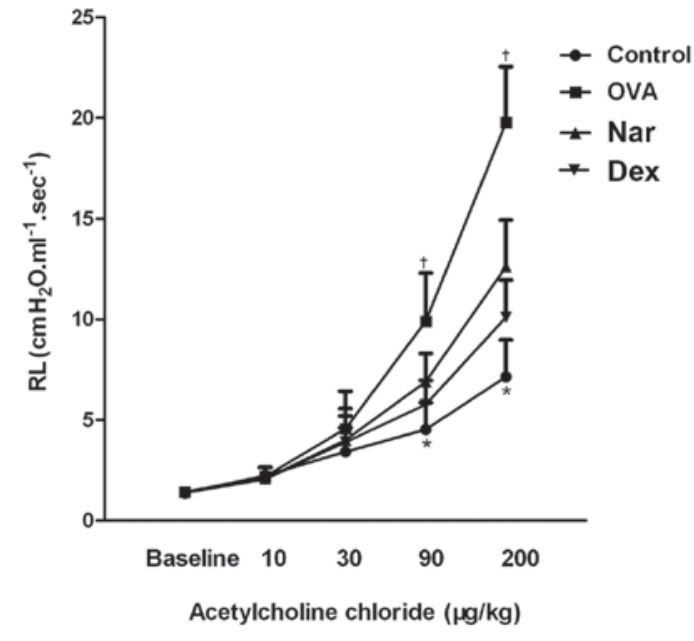

Figure 3. Naringenin abrogates allergen-induced airway hyper-responsiveness in response to methacholine. Results are expressed as the mean resistance of the lung $(\mathrm{RL}) \pm \mathrm{SEM}$ of three independent experiments ( $\mathrm{n}=5 /$ group). ${ }^{*} \mathrm{P}<0.05$ between the control and the ovalbumin (OVA) group; ${ }^{\mathrm{P}} \mathrm{P}<0.05$ between the OVA and dexamethasone (Dex) or naringenin (Nar) groups.

significantly increased compared to those of the control mice. Treatment with naringenin and dexamethasone significantly reduced the number of eosinophils and total inflammatory cells in the BALF (Fig. 2).

Naringenin decreases AHR in a model of chronic asthma. OVA treatment resulted in a marked increase in lung resistance values relative to those of the control group. This increase was reverted by treatment with dexamethasone and naringenin, especially at the 90 and $200 \mu \mathrm{g} / \mathrm{kg}$ methacholine doses (Fig. 3).

Naringenin reduces the level of Thelper 2 (Th2) cytokines in BALF and that of total serum IgE. The levels of Th2 cytokines IL-4 and IL-13 in BALF and that of total serum IgE were markedly increased in the OVA-sensitized and -challenged mice compared to those in the control group. The administration of naringenin and dexamethasone significantly reduced the levels of Th2 cytokines (Fig. 4A and B) in BALF and total serum IgE (Fig. 4C) relative to those in the OVA group.
Naringenin inhibits airway remodeling in a model of chronic asthma. The level of airway metaplasia and mucus production was assessed by PAS staining of lung tissues and the percentage of PAS-positive cells was determined. Overproduction of mucus and goblet cell hyperplasia were observed in the bronchial airways of mice in the OVA group. By contrast, dexamethasone- and naringenin-treated mice showed a reduction in the number of PAS-stained goblet cells (Fig. 5).

The areas of $\alpha$-SMA-stained smooth muscle layers in OVA mice were significantly larger compared to control mice. Administration of naringenin and dexamethasone reduced the areas of $\alpha$-SMA compared to the OVA group (Figs. 5F and 6).

Masson's trichrome staining revealed collagen deposition (fibrosis). The mean area of airway fibrosis in the OVA group was markedly enhanced compared to the control group. Administration of naringenin and dexamethasone caused a reduction in collagen deposition compared to the OVA group (Fig. 7A-E). The total hydroxyproline content in lungs of the OVA group was significantly higher compared to that of the control group. Treatment with naringenin and dexamethasone reduced the hydroxyproline content compared to the OVA group (Fig. 7F).

\section{Discussion}

A previous study showed that naringenin, a naturally occurring flavonoid, attenuates acute airway inflammation and AHR, in association with $\mathrm{NF}-\kappa \mathrm{B}$ repression and reduced cytokine production (9). Consequently, we evaluated the effect of naringenin on airway remodeling using a long-term exposure murine model of asthma. The results of our study clearly demonstrate the inhibitory effect of naringenin on airway remodeling.

Sensitization and chronic challenge of mice with OVA resulted in inflammation of the airway, evidenced here by the recruitment of inflammatory cells, the production of Th2 cytokines and an increase in the total IgE level. The inflammation was effectively attenuated by naringenin treatment. As a natural anti-inflammatory flavonoid, naringenin displayed anti-inflammatory action in a long-term asthmatic process.

Marked goblet cell hyperplasia and mucus hypersecretion were previously observed in the small airway under chronic airway inflammatory conditions (13), consistent with results on our murine model of asthma. In this study, we demonstrated that
A

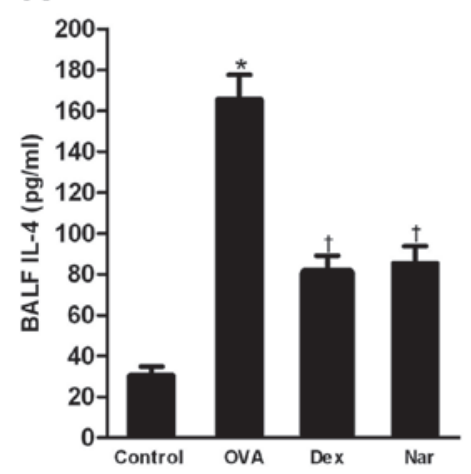

B

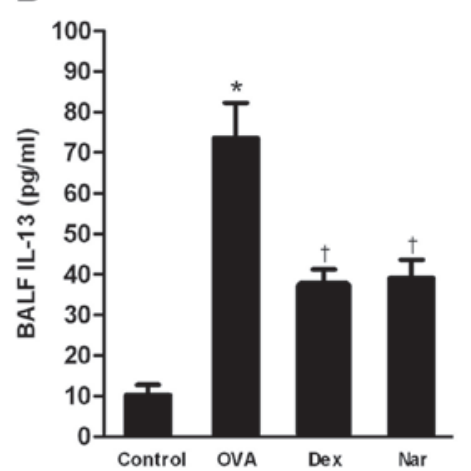

C

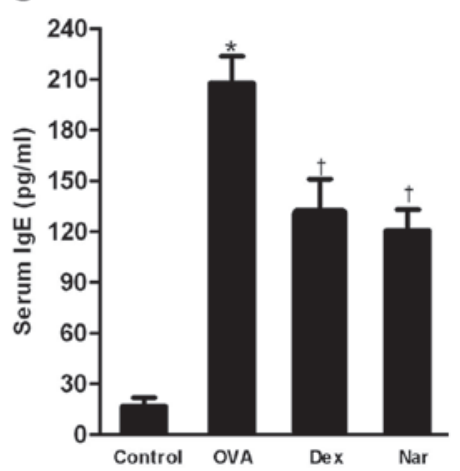

Figure 4. (A) IL-4 and (B) IL-13 levels in bronchoalveolar lavage fluid (BALF), and (C) level of IgE in the serum. Results are presented as means \pm SEM of three independent experiments $(n=6) .{ }^{*} \mathrm{P}<0.05$ between the control and the ovalbumin (OVA) group; ${ }^{\dagger} \mathrm{P}<0.05$ between the OVA and dexamethasone (Dex) or naringenin (Nar) groups. 

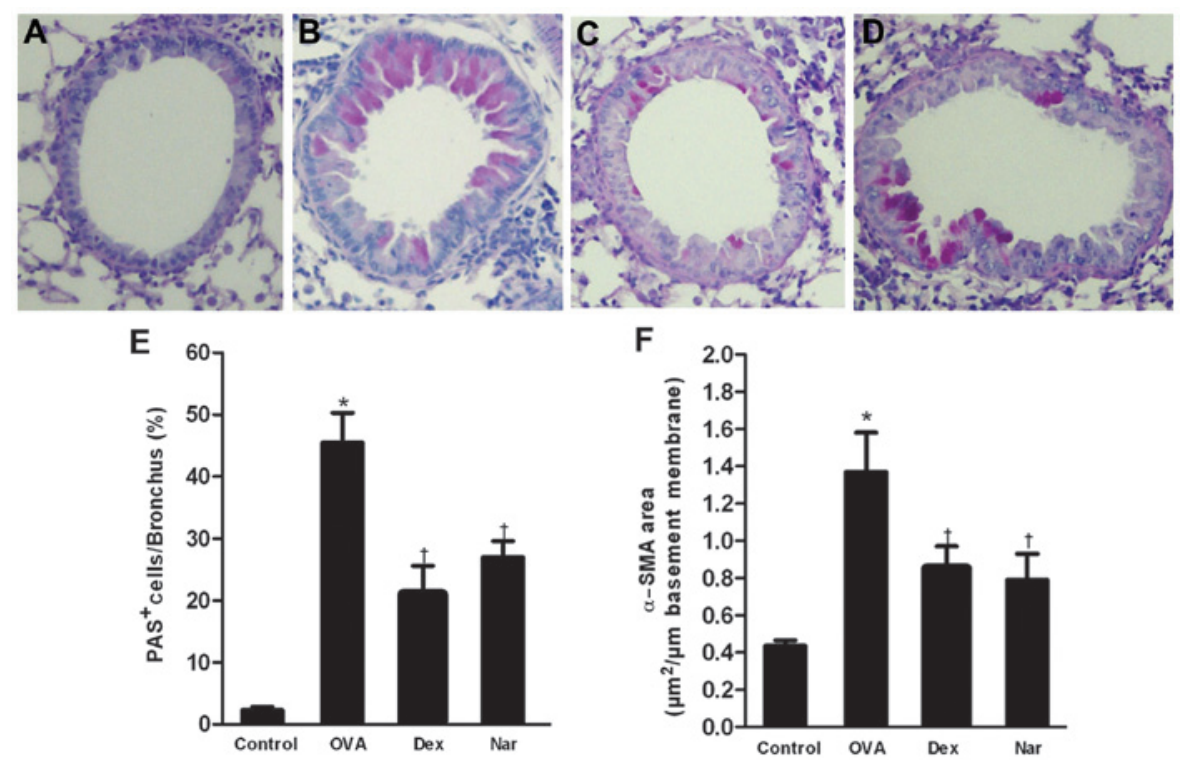

Figure 5. Naringenin attenuates ovalbumin (OVA)-induced mucus production. Images of Periodic-Acid Schiff (PAS) staining (original magnification, x200) in the (A) control, (B) OVA, (C) dexamethasone and (D) naringenin groups. (E) The percentage of PAS-positive cells per bronchiole. (F) The mean areas of peribronchial $\alpha$-smooth muscle actin $\left(\alpha-\right.$ SMA). ${ }^{*} \mathrm{P}<0.05$ between the control and the OVA group; ${ }^{\dagger} \mathrm{P}<0.05$ between the OVA and dexamethasone (Dex) or naringenin (Nar) groups

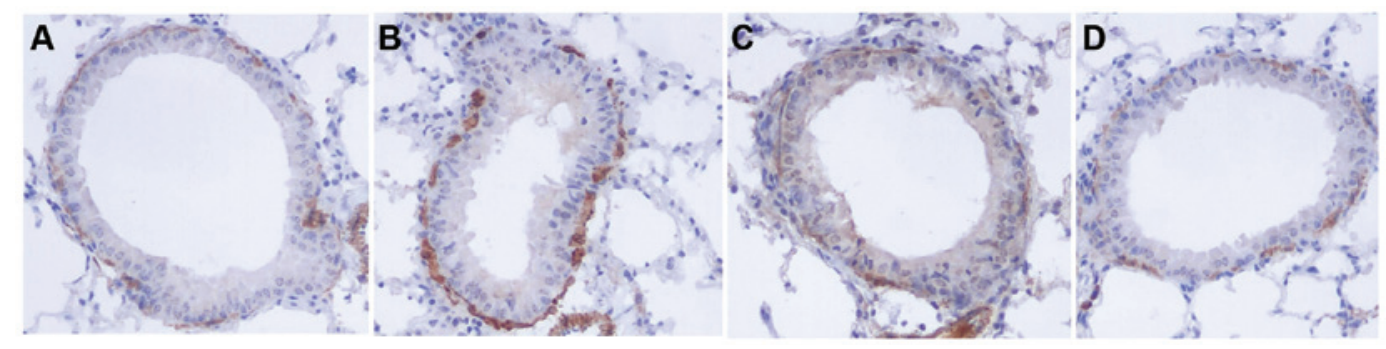

Figure 6. Naringenin treatment reduces the areas of $\alpha$-smooth muscle actin (original magnification, $\mathrm{x} 200$ ). Images of immunohistochemical analyses on the (A) control, (B) ovalbumin (OVA), (C) dexamethasone and (D) naringenin groups.
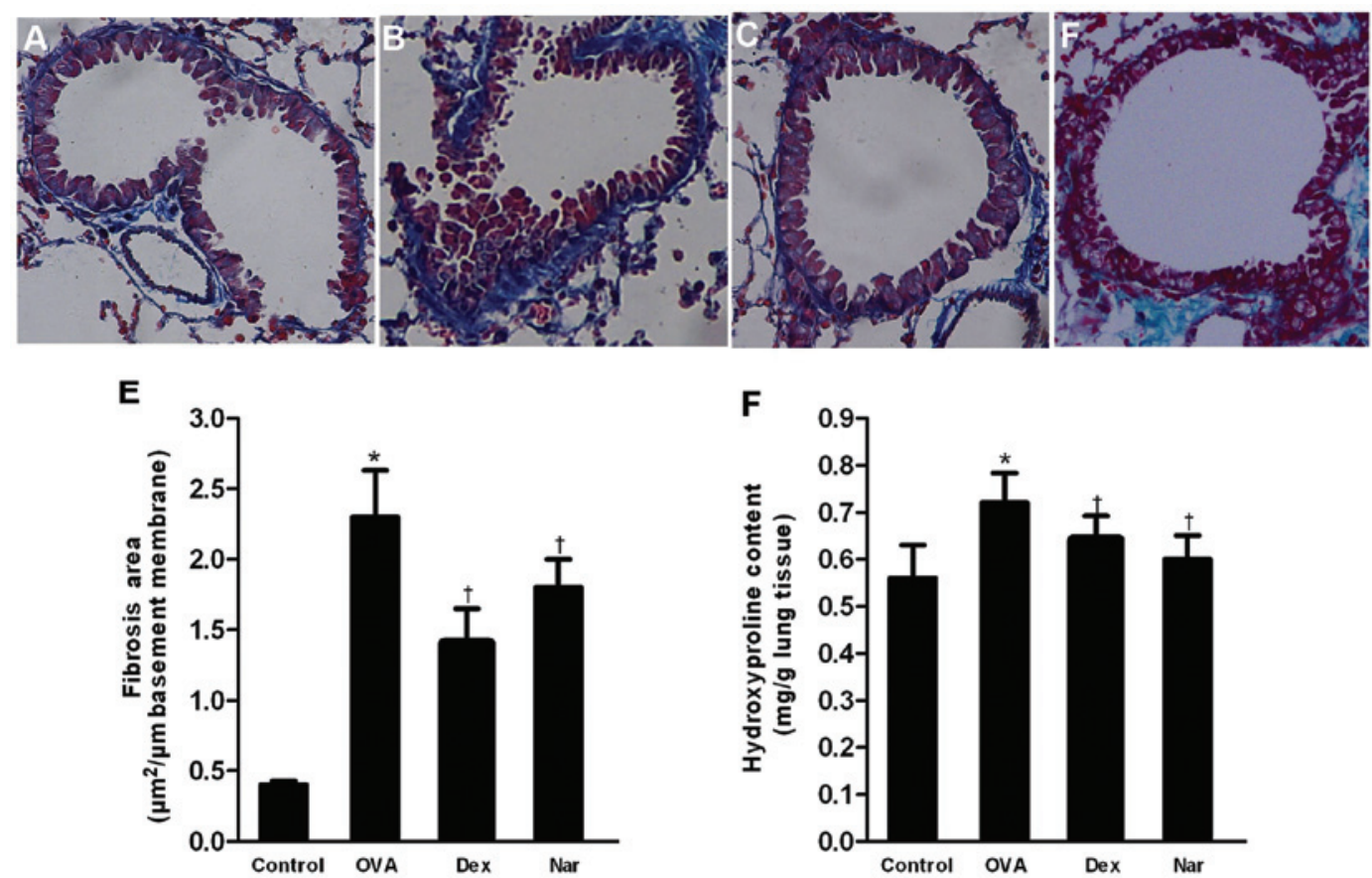

Figure 7. Naringenin inhibits peribronchial fibrosis in a chronic asthma model. Masson's trichrome-positive peribronchiolar collagen layer (original magnification, x200) in the (A) control, (B) ovalbumin (OVA), (C) dexamethasone and (D) naringenin groups. (E) The mean area of airway fibrosis. (F) Hydroxyproline content of the lung. ${ }^{*} \mathrm{P}<0.05$ between the control and the OVA group; ${ }^{ } \mathrm{P}<0.05$ between the OVA and dexamethasone (Dex) or naringenin (Nar) groups. 
naringenin significantly reduced the number of mucus-producing goblet cells within the epithelium after OVA challenge. It has been shown that IL-4 and IL-13 induce goblet cell hyperplasia in the lungs of mice $(14,15)$. Therefore, the ability of naringenin to reduce mucus production in chronic asthma might relate to its inhibitory effect on Th2 cytokine production.

Another important aspect of airway remodeling is airway smooth muscle (ASM) hyperplasia, which has been linked to the severity of asthma (16). Consistent with previous reports, the areas of $\alpha$-SMA were significantly increased after exposure to OVA in our animal model, while ASM hyperplasia was greatly reduced by treatment with naringenin In addition, we found that airway resistance was reduced following naringenin treatment. Since ASM contributes to airway narrowing and AHR in response to a variety of stimuli during asthma, we hypothesize that the ability of naringenin to reduce the thickness of the ASM layer may partially account for the observed decrease in AHR.

Collagen deposition in the airways is also associated with a decrease in lung function and with the severity of asthma in humans. We demonstrated for the first time that naringenin significantly inhibits the deposition of subepithelial collagen in a chronic asthma model. In the asthmatic process, epithelial damage and subsequent Th2-mediated inflammation are expected to cause an increase in the production of a few potent profibrotic cytokines, such as transforming growth factor $\beta$ and tissue inhibitors of metalloproteinases $(17,18)$. Therefore, we assume that the naringenin-mediated reduction in the Th2 cytokine levels may account for the anti-fibrotic activity of naringenin through inhibition of the production of profibrotic cytokines.

Additional mechanisms by which naringenin may modulate airway remodeling exist. Naringenin is a naturally occurring antioxidant (19). Oxidative stress is closely linked to the pathogenesis of chronic airway disorders, such as the increase in microvascular permeability, excessive mucus secretion and remodeling of the extracellular matrix and blood vessels (20). Therefore, naringenin may prevent airway remodeling by reducing the production of reactive oxygen species.

In conclusion, we have shown that treatment with naringenin effectively inhibits allergen-induced airway inflammation and remodeling. The ability of naringenin to modulate chronic asthma pathology renders it a promising target for asthma therapy.

\section{References}

1. Bousquet J, Clark TJ, Hurd S, Khaltaev N, Lenfant C, O'Byrne P and Sheffer A: GINA guidelines on asthma and beyond. Allergy 62: 102-112, 2007.
2. Bousquet J, Jeffery PK, Busse WW, Johnson M and Vignola AM: Asthma. From bronchoconstriction to airways inflammation and remodeling. Am J Respir Crit Care Med 161: 1720-1745, 2000.

3. Vignola AM, Gagliardo R, Siena A, Chiappara G, Bonsignore MR, Bousquet $\mathrm{J}$ and Bonsignore G: Airway remodeling in the pathogenesis of asthma. Curr Allergy Asthma Rep 1: 108-115, 2001.

4. Tagaya E and Tamaoki J: Mechanisms of airway remodeling in asthma. Allergol Int 56: 331-340, 2007.

5. Warner SM and Knight DA: Airway modeling and remodeling in the pathogenesis of asthma. Curr Opin Allergy Clin Immunol 8: 44-48, 2008.

6. Benayoun L and Pretolani M: Airway remodeling in asthma: mechanisms and therapeutic perspectives. Med Sci (Paris) 19: 319-326, 2003 (In French).

7. Park HY, Kim GY and Choi YH: Naringenin attenuates the release of pro-inflammatory mediators from lipopolysaccharide-stimulated BV2 microglia by inactivating nuclear factor- $\mathrm{\kappa} \mathrm{B}$ and inhibiting mitogen-activated protein kinases. Int $\mathrm{J}$ Mol Med 30: 204-210, 2012.

8. Anand K, Sarkar A, Kumar A, et al: Combinatorial antitumor effect of naringenin and curcumin elicit angioinhibitory activities in vivo. Nutr Cancer 64: 714-724, 2012.

9. Shi Y, Dai J, Liu H, et al: Naringenin inhibits allergen-induced airway inflammation and airway responsiveness and inhibits NF- $\kappa \mathrm{B}$ activity in a murine model of asthma. Can J Physiol Pharmacol 87: 729-735, 2009.

10. Cayci C, Wahlquist TC, Seckin SI, et al: Naringenin inhibits neointimal hyperplasia following arterial reconstruction with interpositional vein graft. Ann Plast Surg 64: 105-113, 2010.

11. Chen S, Ding Y, Tao W, Zhang W, Liang T and Liu C: Naringenin inhibits TNF- $\alpha$ induced VSMC proliferation and migration via induction of HO-1. Food Chem Toxicol 50: 3025-3031, 2012.

12. Cho JY, Miller M, Baek KJ, et al: Inhibition of airway remodeling in IL-5-deficient mice. J Clin Invest 113: 551-560, 2004.

13. Rogers DF: Airway goblet cell hyperplasia in asthma: hypersecretory and anti-inflammatory? Clin Exp Allergy 32: 1124-1127, 2002.

14. Kuperman DA, Huang X, Koth LL, et al: Direct effects of interleukin-13 on epithelial cells cause airway hyperreactivity and mucus overproduction in asthma. Nat Med 8: 885-889, 2002.

15. Kuperman DA, Huang X, Nguyenvu L, Holscher C, Brombacher F and Erle DJ: IL-4 receptor signaling in Clara cells is required for allergen-induced mucus production. J Immunol 175: 3746-3752, 2005.

16. Black JL, Panettieri RA Jr, Banerjee A and Berger P: Airway smooth muscle in asthma: just a target for bronchodilation? Clin Chest Med 33: 543-558, 2012.

17. Bosse Y and Rola-Pleszczynski M: Controversy surrounding the increased expression of TGF $\beta 1$ in asthma. Respir Res 8: 66, 2007.

18. Mohamed SA, Noack F, Schoellermann K, et al: Elevation of matrix metalloproteinases in different areas of ascending aortic aneurysms in patients with bicuspid and tricuspid aortic valves. ScientificWorldJournal 2012: 806261, 2012.

19. Yang J, Li Q, Zhou XD, Kolosov VP and Perelman JM: Naringenin attenuates mucous hypersecretion by modulating reactive oxygen species production and inhibiting $\mathrm{NF}-\kappa \mathrm{B}$ activity via EGFR-PI3K-Akt/ERK MAPKinase signaling in human airway epithelial cells. Mol Cell Biochem 351: 29-40, 2011.

20. de Boer WI, Yao H and Rahman I: Future therapeutic treatment of COPD: struggle between oxidants and cytokines. Int J Chron Obstruct Pulmon Dis 2: 205-228, 2007. 